BNL -46279

DE91 015214

\title{
ALGEBRAIC APPROACHES TO NUCLEAR STRUCTURE
}

\author{
Richard F. Casten
}

- Invited Talk Presented at the Conference on

Nuclear Shapes and Nuclear Structure at Low Excitation Energies

Cargese, Corsica, France

June 2-8, 1991 
ALGEBRAIC APPROACHES TO NUCLEAR STRUCTURE

Richard F. Casten

Physics Department

Brockhaven National Laboratory

Upton, New York 11973

Institute for Kernphysik

University of Köln

Köln, Germany

\section{ABSTRACT}

The concept and application of algebraic models to nuclear structure is surveyed with emphasis on interpretations in terms of traditional geometric models. Recent studies relating to the relationship between $\beta$ and $\gamma$ vibrations in deformed nuclei, to the rigidity or sof tness of axial asymmetry, to signature splitting in octupole excitations, to $O(6)$ quantum numbers and multi-phonon excitations, and to pseudo-SU(3) are discussed.

\section{INTRODUCTION}

The last fifteen years have seen a remarkable renaissance in nuclear structure, and a notable part of this has been motivated and mediated through the development and application of a new generation of algebraic or group theoretic models. Such approaches are founded in the geometrical symmetry properties of the nucleus and are appealing because of their power, simplicity, and success. The best known of these today is, of course, the Interacting Boson Approximation (IBA) model, but other approaches, based on pseudo SU(3) and symplectic group theory, are achieving new breakthroughs and, though often less well known, bear careful attention and scrutiny as well. The present discussion is, in no sense, a review of the field (recent overviews already exist ${ }^{1,2}$ ) but rather a short synopsis of some of the basic ideas and results of these models along with a brief summary of some recent work. Most of the emphasis will be on the IBA which is the only sub-area of this growing field. where the author has a chance of adequately conveying a sense of the field. 
Algebraic models share a common approach which is simply described. One starts with a "parent" group embodying, in group theoretic terms, the most general symmetry structure of the system of interest.' This group characterizes all states of this system. Successive subgroups of this group define systems with successively lower symmetry, that is, they can describe particular states or, better, define quantum numbers that act as labels for individual states. Each group, or subgroup, is defined in terms of operators called generators and has associated with it certain other operators, called Casimir operators, that commute with all the generators of the group. If a Hamiltonian, $\mathrm{H}$, is written as a linear combination of Casimirs of a group and subgroups then the eigenvalues can be written, analytically, in terms of the eigenvalues of the Casimirs. In practice, this simply means that one can immediately write down the energies, without diagonalization, and, moreover, $\mathrm{H}$ then describes a system with particular symmetry and, hence, particular transition selection rules (e.g., for E2 transitions) and simple analytic relationships between allowed transitions (e.g., E2 branching ratios). Often, the effects of symmetry breaking (e.g., adding a new term in $\mathrm{H}$ that is not a Casimir operator of the group chain) can be estimated in perturbation theory or by a rather simple diagonalization. In this way, seemingly complex phase transitions can often be treated in terms of the variation of a single parameter.

\section{THE IBA}

Probably the best known current example of this process in nuclear physics is the IBA (refs. 3 and 4). Here, the system is defined in terms of correlated valence nucleon pairs, coupled to total angular momentum 0 or 2 and denoted $s$ and $\mathrm{d}$ bosons, respectively. The degrees of freedom embodied in the model, through the five magnetic substates of the $d$ boson and the one for the $s$ boson, define a 6-dimensional space whose symmetry is denoted by the group U(6). There are three subgroup chains of $U(6)$ that preserve rotational invariance, namely

$\begin{array}{lll} & \mathrm{SU}(3) \supset 0(3) & \mathrm{SU}(3) \\ \mathrm{U}(6) \supset & \mathrm{O}(6) \supset \mathrm{O}(5) \supset \mathrm{O}(3) & \mathrm{O}(6) \\ & \mathrm{U}(5) \supset \mathrm{O}(5) \supset \mathrm{O}(3) & \mathrm{U}(5)\end{array}$

where the labels at the right are the conventional names for these "dynamical symmetries" (dynamical because each describes a system complete with ground and excited states of varying structure).

Geometrically, SU(3) describes a particular kind of symmetric rotor, $\mathrm{O}(6)$ a completely $\gamma$-soft axially asymmetric rotor, and $U(5)$ a spherical vibrator which can be either harmonic or anharmonic (within certain restrictions). Noting that the $\gamma$-instability of $O(6)$ stems from the $O(5)$ subgroup, we recognize that $\mathrm{U}(5)$ is also $\gamma$-unstable. This should not be surprising: a spherical vibrator is trivially $\gamma$-independent. 
It is of the utmost importance to recognize that the IBA explicitly takes account of the finite number, $N_{B}$, of valence nucleons and that, for finite $N_{B}$, these geometrical analogies are only approximate. We shall see implications of this below.

Empirical Manifestations of the Dynamical Symmetries

$1:$

There is no a priori reason that any of these symmetries need exist in real nuclei. It is already sufficient that the IBA structure embodying them provides a powerful means of calculating nuclei (as we shall see) that deviate from the strictures of the pure symmetries. Nevertheless, in fact, nuclei close to each of these symmetries are known. The nucleus ${ }^{196} \mathrm{Pt}$ and those in the $\mathrm{Xe}-\mathrm{Ba}$ region near $A=130$ are excellent examples 5 of $O(6)$. $S U(3)$ is most closely approximated 4,6 in $156 \mathrm{Gd}$ and the $\mathrm{N} \sim 102$ nuclei of $\mathrm{Yb}$ and $\mathrm{Hf}$, while a near harmonic realization of $U(5)$ occurs in the $C d$ isotopes, especially in $118 \mathrm{Cd}$ where mixing with intruder degrees of freedom is minimal ${ }^{7}$. The evidence for each of these has been detailed many times and, hence, rather than repeat it in the limited space here, it is of more interest to focus on a few specific issues of recent importance.

\section{$\beta$ and $\chi$ Vibrations}

In traditional geometrical models, the $\beta$ and $\gamma$ vibrations are completely independent quadrupole excitations of the ground state, generated by $Y_{2,0}$ and $Y_{2, \pm 2}$ operators respectively. The former represents a vibrational extension in deformation (preserving axial symmetry), while the latter corresponds to an oscillation in $\gamma$ (with a $\gamma_{\text {ave }}$ of zero but with finite $\gamma_{\mathrm{rms}}$ ). E2 transitions between $\beta$ and ground bands, or $\gamma$ and ground bands, are allowed and collective, while $\beta \leftrightarrow \gamma$ transitions are forbidden, since they correspond to the creation of one phonon and the destruction of another.

The SU(3) limit of the IBA is radically different. Here, $\beta$ and $\gamma$ bands occur in the same excited representation $[(\lambda, \mu)$ values] which is different from that of the ground state. The E2 selection rule, $\Delta(\lambda, \mu)=(0,0)$ then forbids both $\beta \rightarrow g$ and $\gamma \rightarrow g$ transitions, while $\beta \leftrightarrow \gamma$ transitions are allowed and collective. SU(3) is also a special case of the rotor in that the $\beta$ and $\gamma$ vibrations, again because they occur in the same representation, must be degenerate, $E\left(J_{\beta}\right)=E\left(J_{\gamma}\right)$, for $J_{\beta}=$ $\mathrm{J}$.

Clearly, then, real deformed nuclei, with collective $\beta \rightarrow g$ and, especially, $\gamma \rightarrow g$ transitions in addition to the more recently discovered $\beta \leftrightarrow \gamma$ transitions of collective strength, and with $E_{\beta} \neq E_{\gamma}$, must represent deviations from SU(3). Indeed, the deviations (i.e., the mixing of SU(3) intrinsic excitations in calculations for realistic nuclei) are large, and it is only in the few isolated nuclei listed above where all the SU(3) signatures are approached empirically. What happens in these deviations is of interest. First $\beta \rightarrow g$ and $\gamma \rightarrow g$ E2 transitions become collective ( $\beta \leftrightarrow \gamma$ ones remain so) and, interestingly, the IBA automatically predicts that $B(E 2: \gamma \rightarrow g)>B(E 2: \beta \rightarrow g)$, as observed. Secondly, intraband, $\gamma \rightarrow g$ or $\beta \rightarrow g$, and $\beta \leftrightarrow \gamma$ transitions depend functionally on $N_{B}$ as a 
constant, as $1 / N_{B}$ and as $1 / N_{B}^{2}$, respectively, so that, as $N_{B} \rightarrow \infty$, the traditional results of highly collective intraband transitions, collective $\beta \rightarrow g$ and $\gamma \rightarrow g$ decays and forbidden $\beta \leftrightarrow \gamma$ matrix elements is indeed recovered. For finite and realistic $N_{B}$, however, this is far from realized and experiment clearly supports the IBA scenario.

Linkage between $\beta$-and $\gamma$-vibrations (Collaboration with W.-T. Chou and P. von Brentano)

There is an even more striking consequence of the IBA for the $\beta$ and $\gamma$ band structures. In $\mathrm{SU}(3)$, as noted, these modes are intimately linked. When $\mathrm{SU}(3)$ is broken, as in calculations for realistic deformed nuclei, this linkage is maintained. Specifically, $S U(3)$ is broken by strong $\Delta K=0$, and much weaker $\Delta \mathrm{K}=2$, matrix elements. Thus, the $\mathrm{SU}(3) \beta$ and $\mathrm{g}$ bands mix (and repel). Then, the $\Delta \mathrm{K}=2$ mixing of $\beta$ and $\gamma$ bands leads indirectly to $\gamma-\mathrm{g}$ mixing, that is, to the bandmixing that is so well known in actual deformed nuclei. But this implies that there must be a relationship between $\beta$ and $\gamma$ band properties in deformed nuclei and, specirically, between the energy of the $\beta$ band, relative to the $\gamma$ band, and the decay properties of the $\gamma$ band. Such a relationship is completely foreign to traditional geometric models and unexpected empirically. It has also never been systematically sought in the data. However, it marks a telling test of the IBA and its recognition in the data would revise our understanding of the E2 collective modes of deformed nuclei.

To address this issue, we use the simple IBA Hamiltonian

$$
\mathrm{H}=\mathrm{a}_{2} \mathrm{Q} \cdot \mathrm{Q}
$$

where $Q=\left(s^{\dagger} d+d^{+} s\right)+\chi\left(d^{+} d\right)^{(2)}$

and $\quad T(E 2)=e_{B} Q$

with the same $Q$ operator (same $\chi$ ) as in the Hamiltonian. This is known as the Consistent $Q$ Formalism ${ }^{8}$ or CQF. $a_{2}$ is only a scale factor on energies and is of no consequence here. $e_{B}$ is a boson effective charge and is also of no interest for branching ratios. In $\mathrm{SU}(3) \chi=-\sqrt{7} / 2=-1.32$. $\mathrm{SU}(3)$ symmetry breaking proceeds by changing $\chi$, towards 0 (which gives the $O(6)$ limit). Note that this approach to transition regions is a characteristic one in the IBA, whose "structure" can be viewed in terms of the symmetry triangle of fig. 1. Here, symmetries occupy the vertices, and transition regions the legs. Transit through such a region is then a 1-dimensional (i.e., 1-parameter) problem specified by the position [e.g., $\chi$ value for $S U(3) \rightarrow O(6)$ ] along the leg. For deformed nuclei, which are intermediate between $\mathrm{SU}(3)$ and $\mathrm{O}(6), \chi$ can be fixed from $\gamma$ band E2 decay properties. Then, the $\beta / \gamma$ band energy ratio can be inspected to see if linkage does occur. Since the only relevant parameter is $\chi$, we can see the predicted relation between these observables easily in universal contour plots against $N_{B}$ and $\chi$ such as shown in fig. 2 for several observables involving the $\beta$ and $\gamma$ bands. 

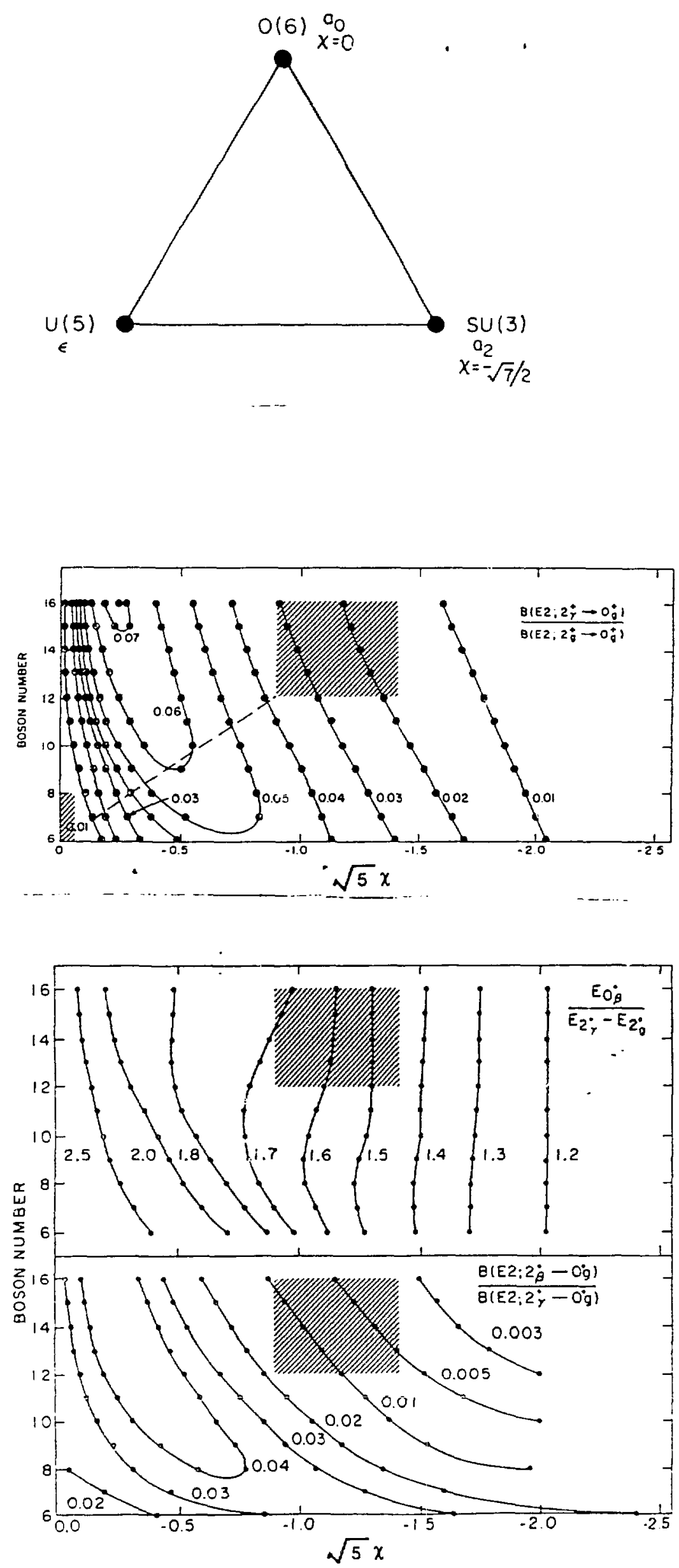

Fig. 1. Symmetry triangle of the IBA. The labels on the vertices refer to the coefficients of the standard IB A multipole

Hamiltonian (see ref. 1) that produce each symmetry.
Fig. 2. Contour plots of the energy ratio of $\beta$ and $\gamma$ bands (top) and of two $B(E 2)$ ratios as functions of $N_{B}$ and $\chi$. (From ref. 8.) 


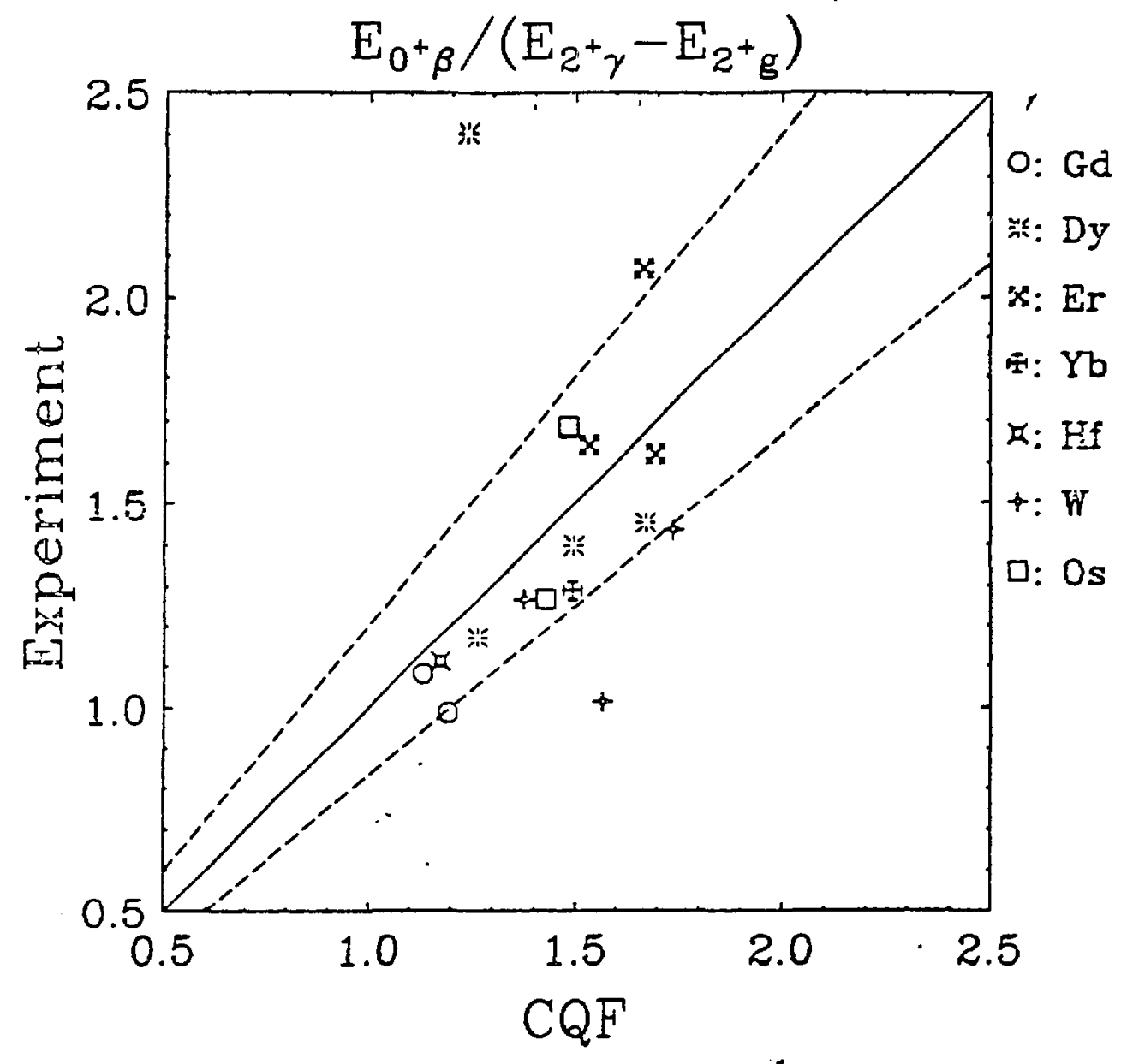

Fig. 3. Plot of experimental vs. theoretical values of $R_{\beta \gamma}$ for CQF IBA calculations. The dashed lines correspond to deviations of $\pm 20 \%$ from perfect agreement.

We are now in a position to inspect the actual data. To do so, we define

$$
\mathrm{R}_{\beta \gamma}=\mathrm{E}_{0_{\beta}^{+}}^{+} /\left(\mathrm{E}_{2}^{+}-\mathrm{E}_{2}^{+}{ }_{\mathrm{g}}^{+}\right)
$$

(This is identical to the ratio in the middle of fig. 2.) We fix $\chi$ from the E2 decay properties of the $\gamma$-band, specifically using empirical values of the bandmixing parameter, $Z_{\gamma}$, and calculate $R_{\beta \gamma}$ and compare with experiment. This is shown ${ }^{9}$ in fig. 3 where $R_{\beta \gamma}$ (IBA) is plotted against $R_{\beta \gamma}$ (Exp): agreement corresponds to points along the $45^{\circ}$ diagonal. While, clearly, perfect agreement is elusive, there is, just as clearly, an evident correlation which goes counter to the prevailing understanding of these excitations and which supports the linkage embodied in the IBA by virtue of the algebraic structure of $S U(3)$ and the way the structure is perturbed en route from $S U(3)$ to $O(6)$. This empirical linkage needs to be understood microscopically. 


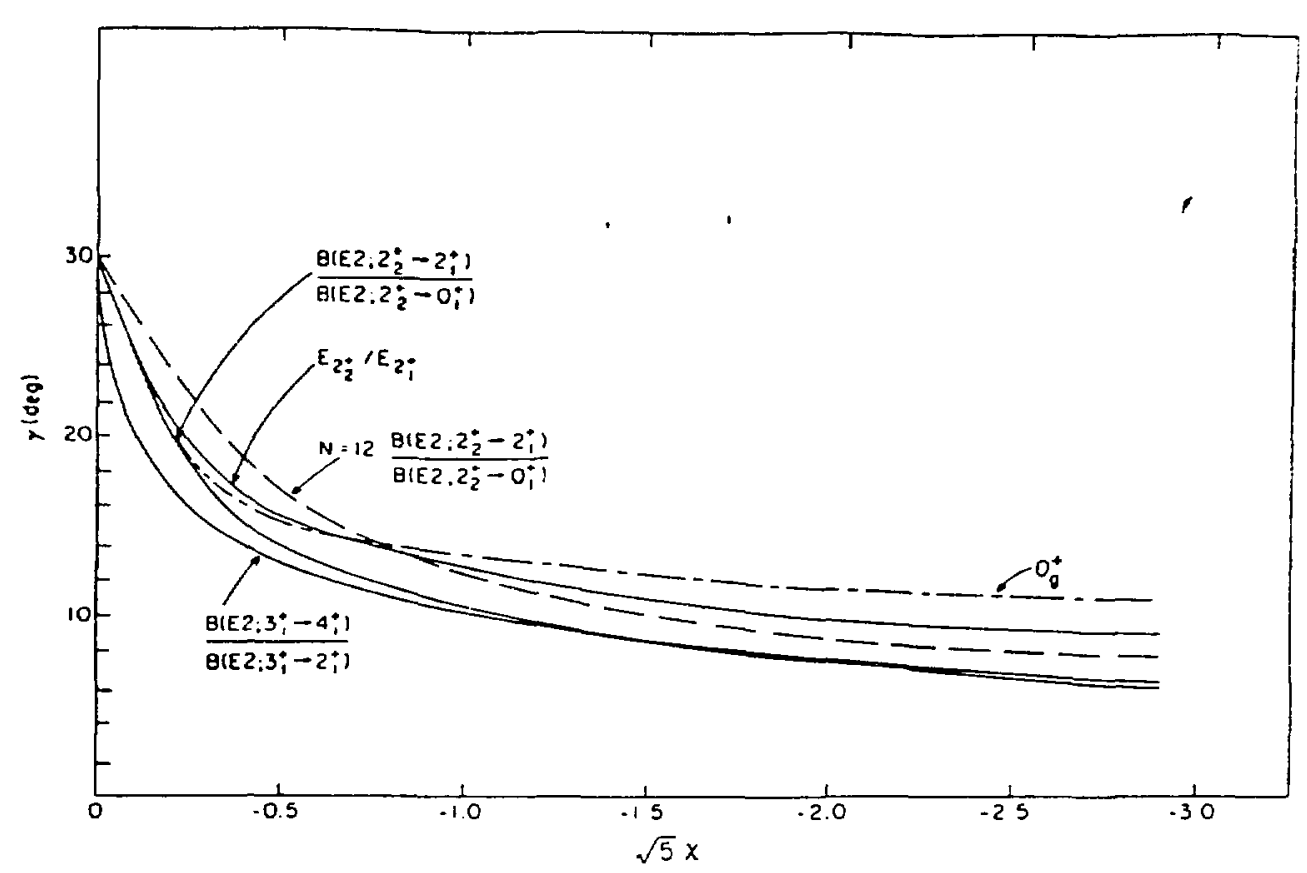

Fig. 4. $\gamma-\chi$ relation in the $C Q F$ (for $N=16$ except where indicated). The curves correspond to different observables. That labelled $0_{\mathrm{g}}^{+}$refers to an analytic shape analysis of the ground state in ref. 12. (Based on ref. 10.)

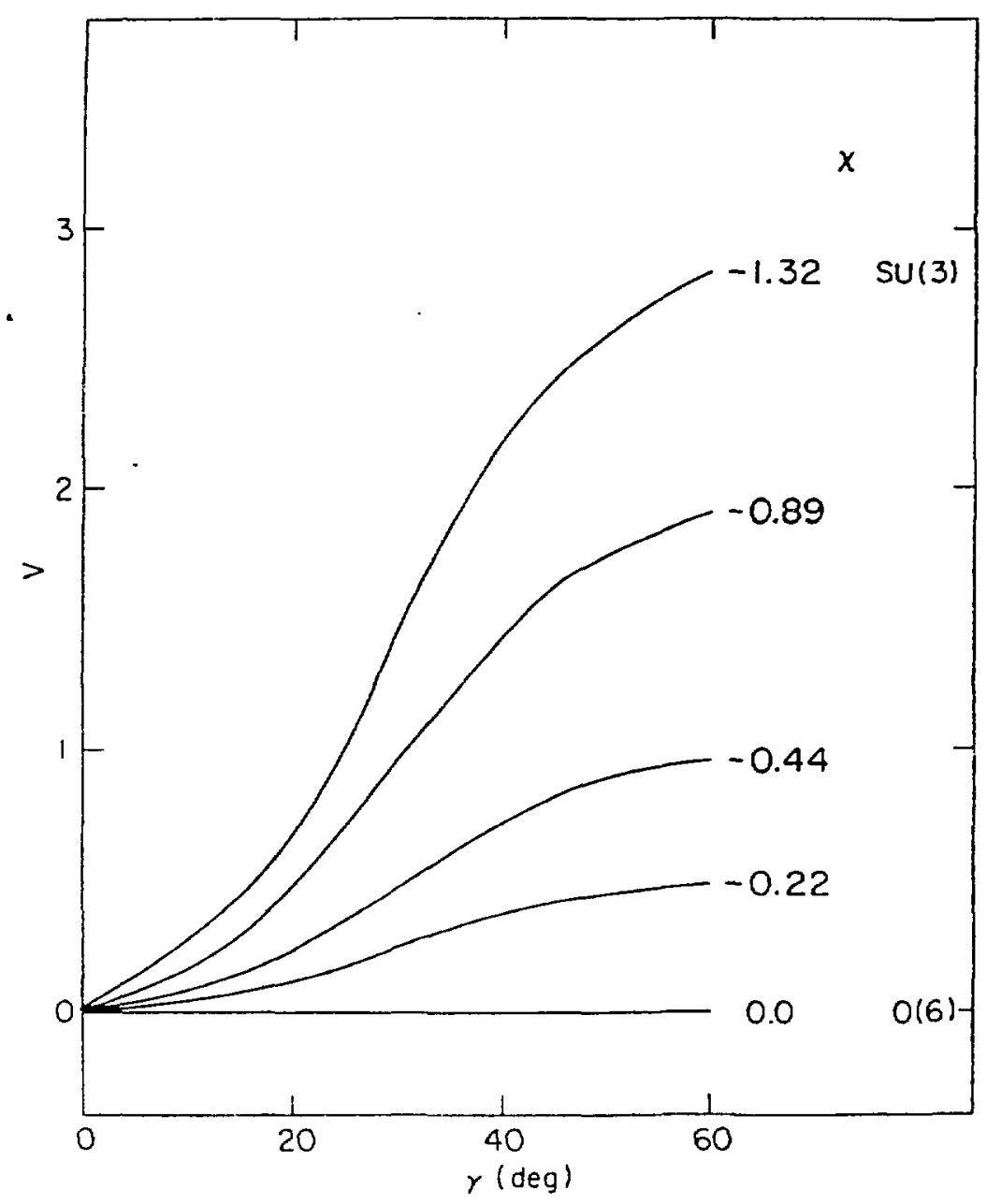

Fig. 5. The IBA potential $V(\gamma)$ as a function of $\gamma$ for several values of $\chi$. (From ref. 10.) 
There is another interesting aspect of an $S U(3) \leftrightarrow O(6)$ region that illustrates nicely the geometrical content of the algebraic IBA. SU(3) is an axially symmetric rotor while $O(6)$ is $\gamma$-unstable with the shape oscillating uniformly from $\gamma=0^{\circ}$ to $60^{\circ}$, giving $\gamma_{\mathrm{rms}}=30^{\circ}$. Hence, somehow, the $\chi=-1.32 \rightarrow \chi=0$ transition leg must correspond to the introduction of axial asymmetry. This has been studied 10 by equating observables calculated as a function of $\chi$ in the IBA to those obtained in the Davydov model ${ }^{11}$ to obtain a $\gamma \leftrightarrow \chi$ correlation. [Even though there is no triaxiality in the IBA, that is, $V(\gamma)$ never has a minimum for $\gamma \neq 0^{\circ}$ and all axial asymmetry is dynamic, the use of the Davydov model in this way has been shown ${ }^{12}$ to be valid.] The results are given in fig. 4. As expected, $\gamma_{\mathrm{rms}}=30^{\circ}$ in $\mathrm{O}(6)$ where $\mathrm{V}(\gamma)$ is flat from $0^{\circ}$ to $60^{\circ}$, and decreases as $\mathrm{SU}(3)$ is approached. Interestingly, $\mathrm{SU}(3)$ corresponds, not to $\gamma$ $=0^{\circ}$ but to $\gamma \sim 10^{\circ}$. The reason is finite $\mathrm{N}_{\mathrm{B}} \cdot \gamma[\mathrm{SU}(3)] \rightarrow 0$ as $\mathrm{N}_{\mathrm{B}} \rightarrow \infty$ but, for finite $N_{B}$, though $V(\gamma) S U(3)$ has a minimum at $\gamma=0^{\circ}$, it is parabolic and finite in $\gamma$ so that $\gamma_{\mathrm{rms}} \neq 0$ (see fig. 5).

Empirical Axial Asymmetry: Soft or Hard? (Collaboration with N. Zamfir)

An on-going issue for decades has been whether empirical axial asymmetry is rigid triaxial or soft. The low lying energy levels of the $\gamma$-band provide a simple and readily available signature that can be inspected, and the IBA, in the CQF, can be exploited ${ }^{13}$ to assess the degree of softness. Rigid triaxial and $\gamma$-unstable models have opposite $\gamma$-band energy staggering as illustrated in fig. 6. The staggering in the Davydov ${ }^{11}$ picture, $\left(2_{\gamma}^{+} 3_{\gamma}^{+}\right),\left(4_{\gamma}^{+} 5_{\gamma}^{+}\right), \ldots .$. arises because, for finite $\gamma$, the even spin $\gamma$-band members mix with the ground band and are repelled upward in energy towards the next higher odd spin level (e.g., $4_{\gamma}^{+}$close to $5_{\gamma}^{+}$). In contrast, for the Wilets-Jean ( $\gamma$-unstable) 14 , or $O(6)$ picture 3 , the levels of ground and $\gamma$-bands are arranged in degenerate multiplets whose origin is related to those of the harmonic vibrator. In the latter, the 1-, 2-, and 3-phonon states multiplets are: $\left(2^{+}\right),\left(0^{+}, 2^{+}, 4^{+}\right)$, $\left(0^{+}, 2^{+}, 3^{+}, 4^{+}, 6^{+}\right)$, respectively. The difference for the $\gamma$-unstable rotor is that the finite $\beta$ (i.e., the rigidity in $\beta$ ) forces the " $\beta$ " band (that is, the $0_{2}^{+}, 2_{\xi}^{+} \ldots .$. ) levels up in energy, leaving behind the Wilets-Jean multiplets $\left(2_{g}^{+}\right),\left(2_{\gamma^{+}}^{+} 4_{g}^{+}\right),\left(0^{+}, 3_{\gamma^{\prime}}^{+}\right.$ $\left.4_{\gamma}^{+}, 6 \mathrm{~g}\right)$, where the $\gamma$ or $g$ nature has been labelled where appropriate. Hence, in the $\gamma$-band, each even spin level is associated with the next lower odd spin state (e.g., $4_{\gamma}^{+}$with $3_{\gamma}^{+}$).

To assess the data, we use the signature ${ }^{15}$

$$
S(J, J-1, J-2)=\frac{\left(E_{J}-E_{J-1}\right)-\left(E_{J-1}-E_{J-2}\right)}{E_{2}^{+}}
$$

where the energy levels in the numerator are those of the $\gamma$-band. A rigid triaxial rotor has positive $S$ (for example, $S(4,3,2)=+1.67$ for $\gamma=30^{\circ}$ ) while the $\gamma$-unstable rotor has negative $S(S(4,3,2)=-2)$. The data for $S(4,3,2)$ and $S(6,5,4)$ are displayed in fig. 7 and compared 13 with several models, in particular the 


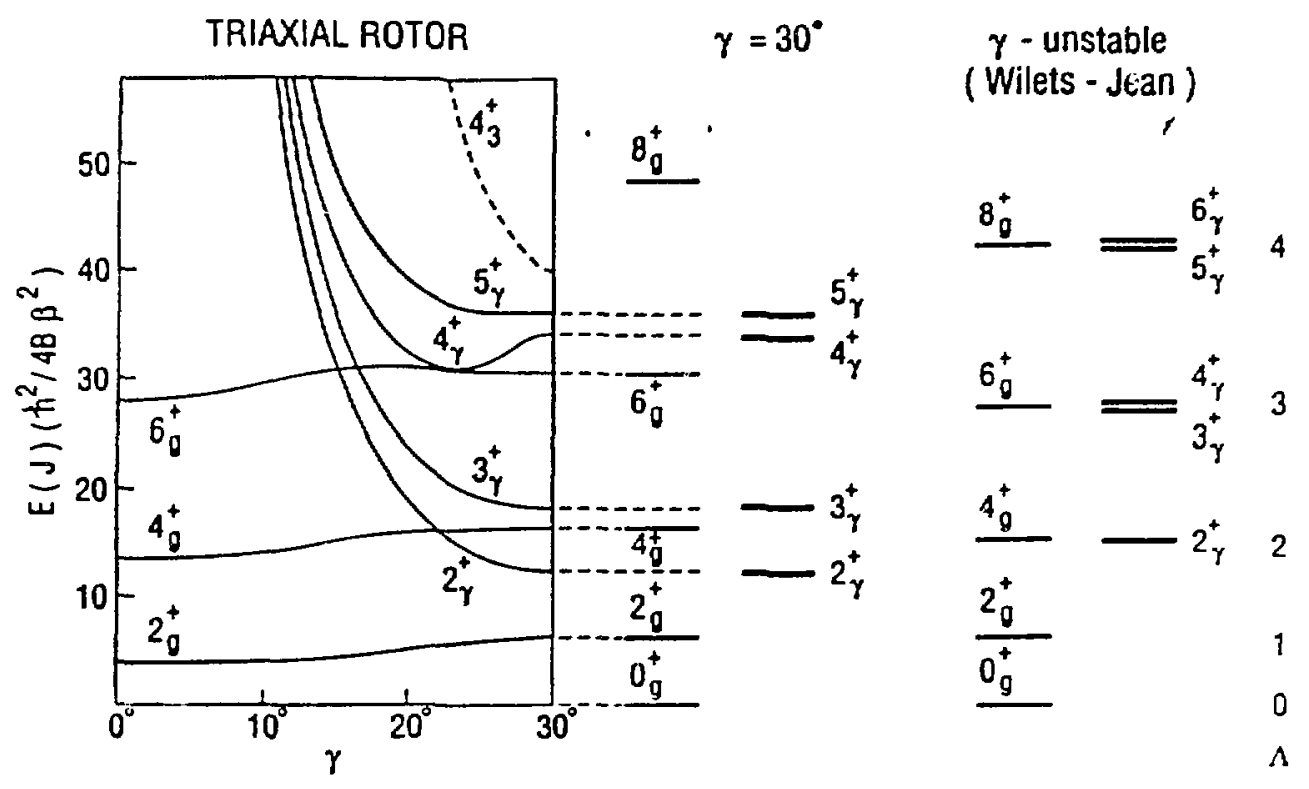

Fig. 6. Comparison of the Davydov rigid triaxial rotor and Wilets-Jean or $\gamma$ unstable rotor pictures. The Davydov results for $\gamma=30^{\circ}$ are shown explicitly near the middle for easier comparison. The $\gamma$-band levels are given as thicker lines. (From ref. 13.)

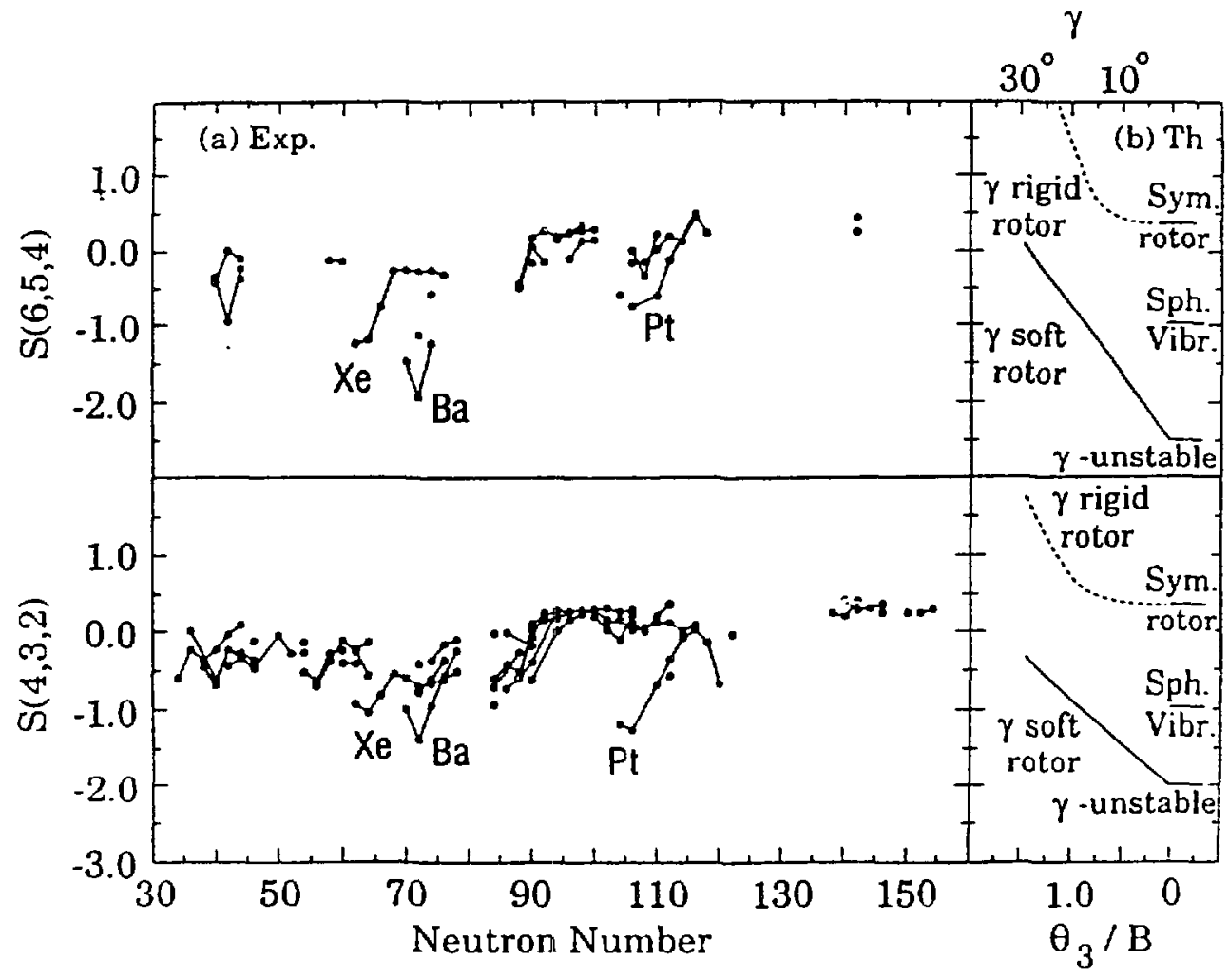

Fig. 7. $\gamma$-band energy staggering signatures $S(4,3,2)$ and $S(6,5,4)$ for $N>30$. On the right are the predictions of several models. (From ref. 13.) 
Davydov model as a function of $\gamma$, and $O(6)$. Clearly, all the data fall either very close to the symmetric rotor value (hardly surprising for well deformed regions) or below it in the direction of $\gamma$-softness, especially in nuclei such as $\mathrm{Xe}, \mathrm{Ba}, \mathrm{Pt}$ which have already been described 5 as $\mathrm{O}(6)$-like. Now ${ }_{4}$ to understand these results, we need to know how $S^{\prime}$ changes with the introduction of $\gamma$ dependence in the potential. To do this, we exploit a simple extension to the IBA in which a cubic term [of the form $\left.H_{\text {cubic }}=\left\{\left(d^{+} d^{+} d^{+}\right)^{(3)}(d d d)^{(3)}\right\}^{(0)}\right]$ is introduced 16 . This adds a term to the potential $V(\gamma)$ with a minimum at $\gamma=$ $30^{\circ}$. We take

$$
\mathrm{H}=\mathrm{H}[0(6)]+\theta_{3} \mathrm{H}_{\text {cubic }}
$$

The energy spacings in the $\gamma$-band, in $O(6)$, are proportional to a parameter $B$ contained in $\mathrm{H}[\mathrm{O}(6)]$. Hence the degree of $\gamma$-dependence is given simply by $\theta_{3} / B$. Large values of $\theta_{3} / B$ reduce the typical $O(6)$ or Wilets-Jean $\gamma$-band energy staggering, in the direction of a triaxial rotor. The question is, how much $\gamma$ dependence is needed to change $S$ by a given amount? The answer is startling, as shown by the line on the lower right in fig. 7 giving calculated values of $S$ (for $N_{B}=6$ ) for several $\theta_{3} / B$. It is seen that values of $\theta_{3} / B$ as small as unity can account for the data. However, $\theta_{3} / B=1$ corresponds to a minimum in $V(\gamma)$ at $\gamma=30^{\circ}$ which, for $N_{B}=6$, amounts to only $69 \mathrm{keV}$ (it is somewhat larger for larger $N_{B}$ ). This $\gamma$-dependence can be expressed by a $\delta \mathrm{V} / \mathrm{V}$ ratio of $2.2 \%$ where $\delta V$ is the maximum change in $V(\gamma)$ induced by the finite $\dot{\theta}_{3}$ for $\theta_{3} / B=1$. Thus, we reach two striking conclusions ${ }^{13}$. First, real nuclei with large asymmetry are extremely $\gamma$ soft, and, secondly, $S$ values between $O(6)$ and Davydov, do not at all correspond to potentials intermediate in $\gamma$-dependence between $\gamma$-flat and $\gamma$-rigid: $S$ is an extremely sensitive indicator of even very weak $\gamma$-dependence.

\section{Octupole Excitations and the IBA (Collaboration with N. Zamfir,}

P. von Brentano, F. Seiffert, and W. Lieberz)

In the $U(5)$ symmetry, the levels of the quasi- $\gamma$-band can be described in terms of the weak coupling of a quadrupole excitation to the yrast states. This is consistent with the $\gamma$-band staggering: to make either a $3_{\gamma}^{+}$or $4_{\gamma}^{+}$excitation requires the coupling $2_{\mathrm{g}}^{+} \otimes 2_{\gamma^{\prime}}^{+}$while both $5_{\gamma}^{+}$and $6_{\gamma}^{+}$states are of the form $4_{\mathrm{g}}^{+} \otimes$ $2_{\gamma}^{+}$. Although it is not as intuitively obvious, the " $\gamma$-band" in $O(6)$ is also a quadrupole excitation weakly coupled to the ground state band. (This is as clear from the wave functions as in $U(5)$ when $N_{B} \rightarrow \infty$.)

This leads to an interesting prediction. Suppose one asks what energy staggering would be expected of an octupole or f-boson excitation in an axially asymmetric nucleus. If the asymmetry is rigid (Davydov rotor), the $\gamma$-band, like the ground band, is a complex mixture of $\mathrm{K}=0$ or 2 components and provides no particular clue: detailed calculations of the negative parity states would be required for an answer. The weak coupling character of the $\gamma$-band in a $\gamma$-soft nucleus, though, provides an immediate intuitive expectation: a weakly coupled f-boson, with $l=3$, should have energy staggering exactly analogous to that of the $\gamma$-band, except shifted upwards one unit in spin (i.e., the staggering would be out of phase with that of the $\gamma$-band): each even spin 

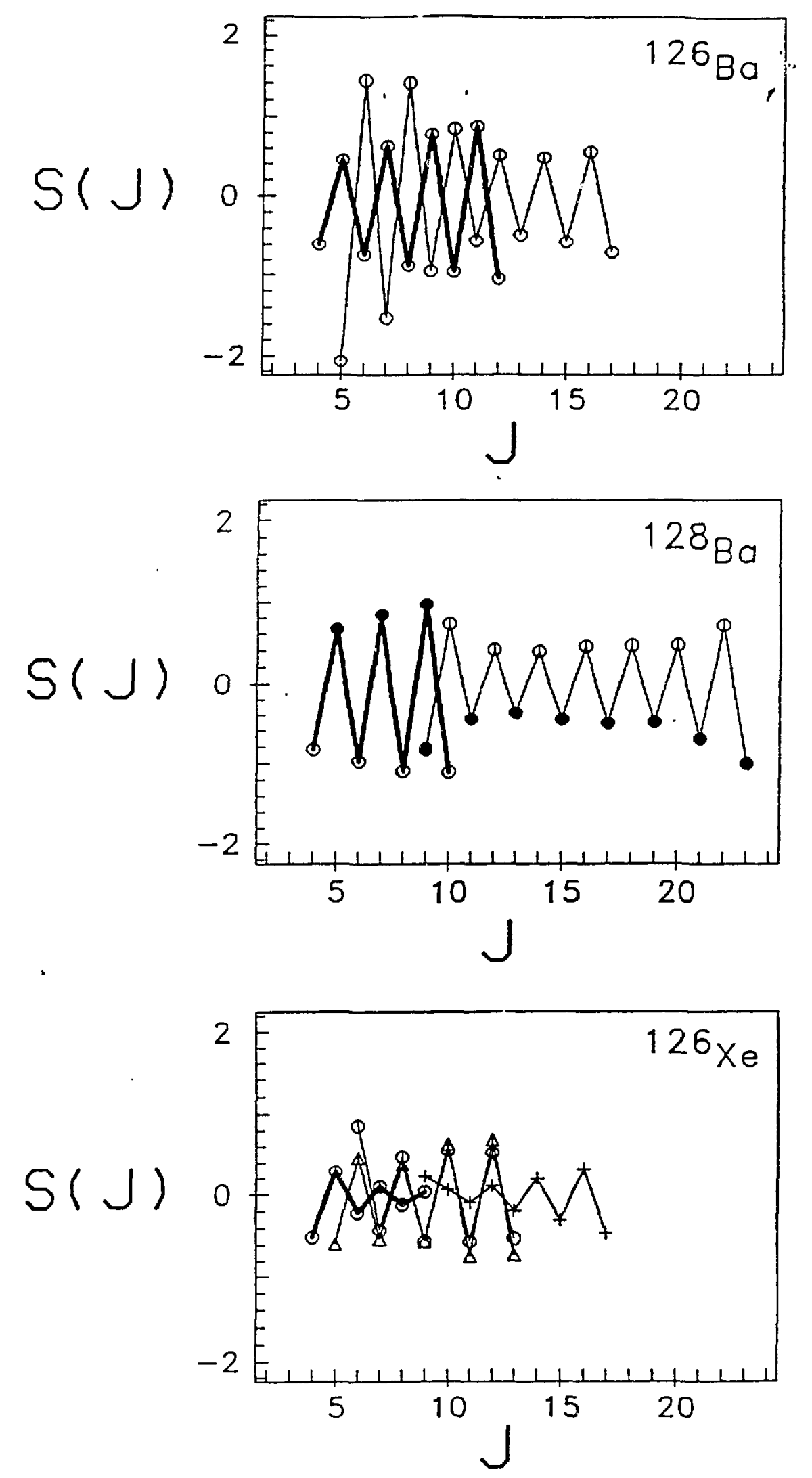

Fig. 8. $S(J)$ plots for several axially asymmetric nuclei near $A=130$. The thick lines are for the $\gamma$-bands, the thin ones for negative parity bands. (From ref. 19.) In the middle panel the data for J odd are marked by solid dots to highlight the fact that $S\left(J_{\text {odd }}\right)$ is a maximum for the $\gamma$ band and a minimum for the negative parity bands. 
level would be degenerate with the next higher, rather than lowsr, odd spin state.

This simple prediction can be tested since there is much fecent medium and high spin data in the $A=13 \dot{0}, \mathrm{Ba}$ and $\mathrm{Xe}$ nuclei ${ }^{17}$. To do this, we utilize another staggering index $S(J)$, which is slightly different, but analogous, to $S(\psi \mathrm{J})$ $1, \mathrm{~J}-2)$ defined earlier. $S(\mathrm{~J})$ is defined ${ }^{18}$ by

$$
S(J)=\frac{R\left(E_{j}\right)}{R\left(E_{j}\right)_{\text {rotor }}}-1
$$

where $\quad R\left(E_{j}\right)=\frac{2\left[E_{J}-E_{J-1}\right]}{E_{J}-E_{J-2}}, \quad R\left(E_{J}\right)_{\text {rotor }}=\frac{J}{J-\frac{1}{2}} \sim 1$

For the $\gamma$ band in $\mathrm{O}(6)$, and $\mathrm{J}_{\mathrm{odd}}$, we have $\mathrm{E}_{\mathrm{J}-1} \sim \mathrm{E}_{\mathrm{J}-2}$ so that $\mathrm{R}\left(\mathrm{E}_{\mathrm{J} \text { odd }}\right) \sim 2$. Hence, $\mathrm{S}\left(\mathrm{J}_{\text {odd }}\right)_{\gamma} \sim+1$. Analogously, $\mathrm{S}\left(\mathrm{J}_{\text {even }}\right)_{\gamma}=-1$. Our expectation is that $\mathrm{S}(\mathrm{J})$ will have the opposite phase for an octupole or single f-boson excitation, that is $\mathrm{S}\left(\mathrm{J}_{\text {even }}\right)_{\text {oct }} \sim+1, \mathrm{~S}\left(\mathrm{~J}_{\mathrm{odd}}\right)_{\text {oct }}=-1$. [This prediction is verified by detailed calculations with the Hamiltonian $\left.\mathrm{H}=\mathrm{H}[\mathrm{O}(6)]+\varepsilon \mathrm{f}^{\dagger} \mathrm{f}+\mathrm{kQ} \cdot \mathrm{Q}_{\mathrm{f}}\right]$ The data for several $A \sim 130$ nuclei are summarized ${ }^{19}$ in fig. 8 and show precisely this feature. These data represent the first clear evidence of the effects of an $O(6)-f$ boson coupling scheme and highlight another illustration of a situation where traditional models do not provide an intuitive solution, and detailed calculations are difficult and not yet practical, but where an algebraic approach gives an immediate prediction, verified by simple calculations, and in agreement with experiment.

\section{Quantum Numbers of $O(6)$}

There are two quantum numbers (besides the total spin $\mathrm{J}$ ) that characterize $O(6)$, namely $\sigma$, which labels different representations of the subgroup $O(6)$, and $\tau$, which labels levels within an $O(6)$ representation according to $O(5)$ and which resembles a phonon-like quantum number. The E2 selection rules are $\Delta \tau= \pm 1$ and $\Delta \sigma=0$. The first has been thoroughly tested as have the predictions of particular branching ratios and absolute $B(E 2)$ values within a given $\sigma$ representation. However, although several levels have been assigned ${ }^{5}$ to excited $(\sigma<N)$ representations in ${ }^{196} \mathrm{Pt}$, and branching ratios are reasonable, there has not been, until recently, any quantitative test of the $\sigma$ quantum number. What is needed is an absolute $B(E 2)$ value that violates the $\Delta \sigma=0$ rule. If $O(6)$ is a reasonable approximation, this $B(E 2)$ value should be much less than allowed $B(E 2)$ values.

Unfortunately, such $B(E 2)$ values, for high lying states weakly connected to the lower levels, are extremely difficult to measure with techniques such as Coulomb excitation or in decay experiments. 
However, this issue has been recently addressed and solved 20 using a new technique, called GRID, in which the miniscule Doppler broadening, from decay in flight, of a nucleus recoiling from prior emission of a $\gamma$-ray, is observed. This remarkable technique ${ }^{21}$, developed and installed at the ILL in Grenoble, France, is described in another contribution 22 to these proceedings by Jan Jolie. [That paper ${ }^{22}$ also discusses other recent GRID experiments, including one 23 that established proof of the collectivity of 2-phonon excitations in deformed nuclei, and another measurement, in progress, of the collectivity of possible 3-phonon states in spherical nuclei.] GRID accesses level lifetimes shorter than a few ps. In an $\mathrm{O}(6)$ nucleus like ${ }^{196} \mathrm{Pt}$ the expected lifetime for the decay of the "bandhead" of the excited $0^{+}$state with $\sigma=\mathrm{N}-2=4$ at $1402 \mathrm{keV}$, would be $\sim 0.2 \mathrm{ps}$ if the $0^{+} \rightarrow 2_{1}^{+} \mathrm{E} 2$ transition were collective. The measured ${ }^{24}$ value, $\tau_{1 / 2}(1402)>1.86 \mathrm{ps}$ is an order of magnitude larger. Since this is a conservative limit, it establishes that the $B\left(E 2: 0_{1402}^{+}(\sigma=4) \rightarrow 2_{355}^{+}(\sigma=6)\right)$ is at least an order of magnitude smaller than for allowed transitions in $O(6)$ and establishes the goodness of the $\sigma$ quantum number.

\section{PSEUDO-SU(3) AND SYMPLECTIC MODELS}

The IBA is a valence space boson model. Algebraic techniques can also be applied directly to fermion excitations (e.g., Elliott $25 \mathrm{SU}(3)$ ) and, in symplectic models, need not be limited to a single shell. Such approaches are at once more microscopic but also generally more complicated. 'Despite the added complexity, important recent breakthroughs have been achieved. Two are worth citing.

The SU(3) symmetry that has been successful25 in light nuclei runs into difficulties in heavier species because one orbit from each shell (the jmax $=\mathrm{N}+$ $1 / 2$ orbit) is pushed down in to the next lower shell by the $l^{2}$ and $l \cdot s$ forces. This badly breaks the oscillator symmetry and precludes the use of SU(3) in such mass regions. This difficulty can be circumvented by the so-called pseudo-SU(3) scheme ${ }^{26}$. Here, briefly, a set of orbits, such as the positive parity states of the 50-82 shell $s_{1 / 2}, d_{3 / 2}, d_{5 / 2}, g_{7 / 2}$ (with $l=0,2,4$ but lacking the $g_{9 / 2}$ orbit) is treated as if they comprised a pseudo-(N=3) shell with $j=1 / 2,3 / 2,5 / 2$, $7 / 2$, constructed from pseudo $l=1,3$ and a nuclear spin of $1 / 2$. The unique parity $1 h_{11 / 2}$ orbit in the $50-82$ shell is treated passively, generally with pair occupation in seniority zero configurations. The advantage of pseudo-SU(3) stems from the following point. In the normal shell model Hamiltonian, the $\bar{l} \cdot \bar{s}$ (and $\bar{l}^{2}$ ) terms break SU(3). However, when the pseudo-spin transformation is made, most of the effects of the $\vec{l} \cdot \bar{s}$ term are incorporated into the new Hamiltonian. The quadrupole-quadrupole interaction that is added in the Nilsson model nearly preserves pseudo-SU(3) and therefore, now, the only major pseudo-SU(3)-breaking term is $l^{2}$ which is overwhelmed by the Q.Q interaction for large deformation. When this happens the pseudoSU(3) quantum numbers, and associated selection rules, decoupling parameters, and so on, become excellent approximations. It thus turns out that the pseudo-SU(3) scheme gives an excellent treatment of the normal parity levels of the Nilsson model in medium and heavy nuclei and, in fact, gives asymptotic predictions that are closer to those of Nilsson wave functions 

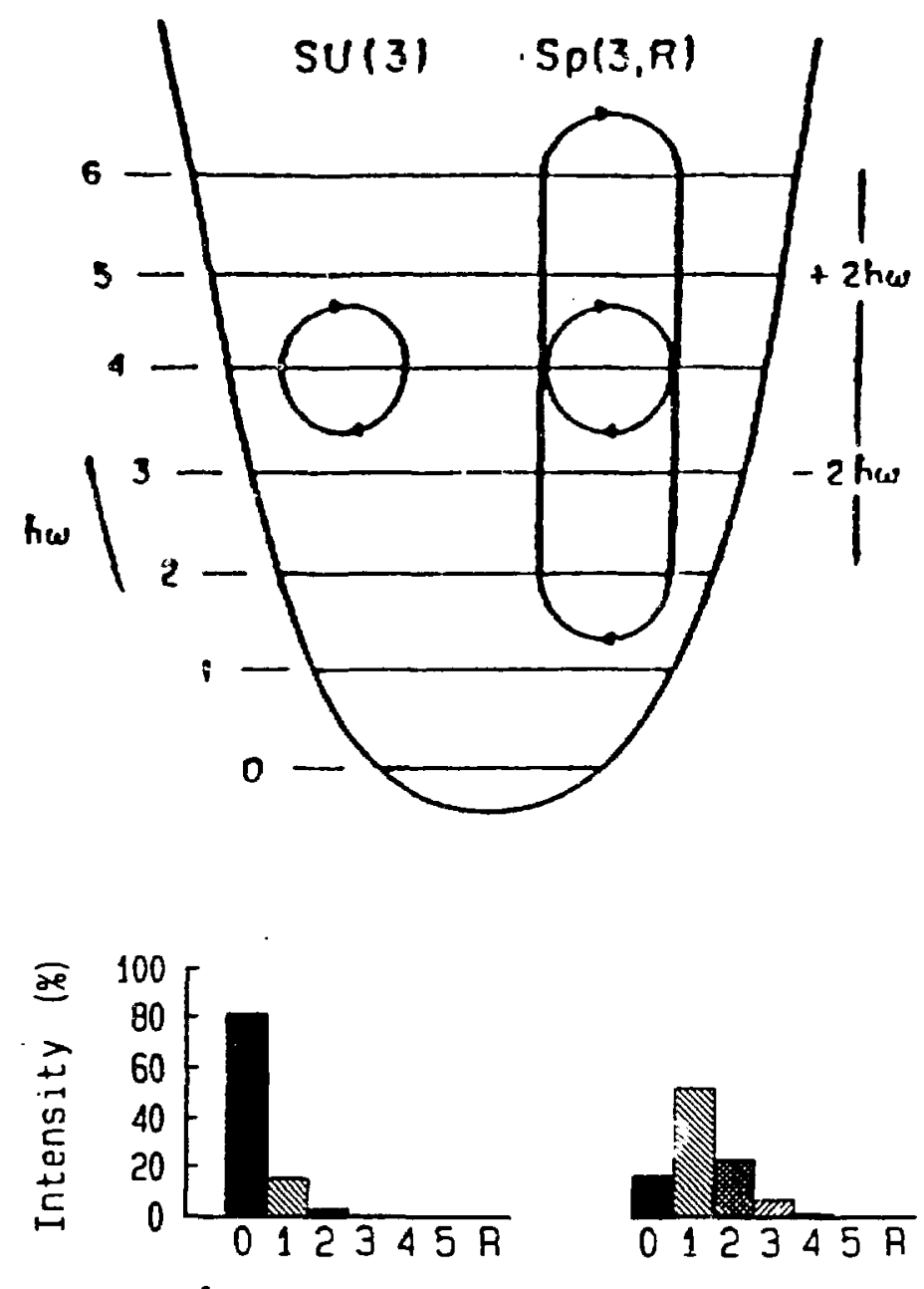

Fig. 9. (Top) Schematic illustration of the differences between the normal $S U(3)$ approach and the $S p(3, R)$ symplectic theory. (Based on the second of ref. 27.) (Bottom) Example of the \% distribution of wave function amplitudes over different shells for a symplectic model calculation of the ground state (G.S.) and E2 giant resonance state (R.S.) of $238 \mathrm{U}$. The abscissa labels the number of $2 \hbar \omega$ boson excitations. (Based on the first of ref. 31.)

for realistic deformation than are the asymptotic Nilsson wave functions themselves. The reader is referred to ref. 27 for elegant summaries of the basic ideas of pseudo-SU(3).

One of the predictions of pseudo-SU(3) is that $K=1 / 2$ bands have decoupling parameters of $a= \pm 1$. Whether this prediction is taken alone 28 , or embodied in a supersymmetry (SUSY) scheme ${ }^{29}$ via the IBA and IBFA, it can directly give predictions in accord with recent observations 30 of the so-called "identical superdeformed bands" in adjacent even and odd mass nuclei.

The second illustration embodies pseudo-SU(3) into a multi-shell (multi$\hbar(\omega)$, or "vertical", space, treated by exploiting the properties of symplectic groups such as $S p(3, R)$. This approach, which is the subject of the excellent recent reviews in refs, 2,27 and 31 . has the anmonlina forturn that it 
simultaneously incorporates intra- and inter-shell $\mathrm{B}(\mathrm{E} 2)$ calculations, that is, it is capable of simultaneously treating the collectivity of $\mathrm{E} 2$ giant resonances and low lying quadrupole collectivity on the same unified footing.

The multi-h $\omega$ approach is illustrated schematically at the top $6 \mathrm{f}$ fig. 9. The lower part of the figure shows a typical recent example of an application of the pseudo-symplectic model to a heavy deformed nuclei, ${ }^{238} \mathrm{U}$. While the ground state is comprised predominantly of $0 \hbar \omega$ components, $2 \hbar \omega$ excitations are not at all negligible. Even more dramatic is the structure of the E2 giant resonant state (R.S.) which contains major amplitudes beyond the traditional description in terms of $2 \hbar \omega$ excitations, including amplitudes for $0 \hbar \omega$ and extending to $12 \hbar \omega$. Work in fermionic algebraic models such as this is now breaking new ground and achieving important results which auger well for the future of this approach and which offer the hope of a group theoretical avenue to understanding the microscopic structure of rotational motion and quadrupole collectivity.

\section{CONCLUSIONS}

In these few pages we have tried to outline some of the features of algebraic approaches to nuclear structure, both in their pure algebraic or symmetry limits and when used as a starting point for symmetry breaking calculations. Emphasis has been on the geometric content of the different symmetries and on their relationships to other collective models. Results focussed on the relation of $\beta$ and $\gamma$ vibrations in deformed nuclei, on the softness of axially asymmetry in nuclei, on signature splitting in octupole excitations of axially asymmetric nuclei, on recent tests of the $\sigma$ quantum number of $O(6)$, on pseudo-SU(3) and identical superdeformed bands in adjacent odd and even nuclei, and on symplectic approaches to the quantum rotor.

\section{ACKNOWLEDGEMENTS}

I am grateful to my collaborators in much of this work, in particular to W.T. Chou, N. Zamfir, A. Aprahamian, D. D. Warner, P. von Brentano, J. A. Cizewski, A. Gelberg, F. Seiffert, W. Lieberz, H. Börner, and J. Jolie. For work which is not yet published, these collaborators are explicitly noted in the tex. In addition, many useful discussions with K. Heyde, D. S. Brenner, I. Talmi, F. Iachello, J. P. Draayer, J.-Y. Zhang, and W. Nazarewicz are thankfully acknowledged. Work has been supported by the United States Department of Energy under contract No. DE-AC02-76CH00016, and by the BMFT.

\section{REFERENTCES}

1. R. F. Casten and D. D. Warner, Rev. Mod. Phys. 60:389 (1988).

2. J. P. Draayer, Nucl. Phys. A520:259c (1990).

3. A. Arima and F. Iachello, Phys. Rev. Lett. 35:1069 (1975).

4. F. Iachello and A. Arima, "The Interacting Boson Model," Cambridge University Press, Cambridge (1987). 
5. J. A. Cizewski et al., Phys. Rev. Lett. 40:167 (1978); R. F. Casten and P. von Brentano, Phys. Lett. 152B:22 (1985):

6. R. F. Casten, P. von Brentano, and A.M.I. Haque, Phys. Rev. C31:1991 (1985).

7. A. Aprahamian et al., Phys. Rev. Lett. 59:535 (1987).

8. D. D. Warner and R. F. Casten, Phys. Rev. Lett. 48:1385 (1982); Phys. Rev. , C28:1798 (1983).

9. W.-T. Chou, R. F. Casten, and P. von Brentano, to be published.

10. R. F. Casten, A. Aprahamian, and D. D. Warner, Phys. Rev. C29:356 (1984).

11. A. S. Davydov and G. F. Filippov, Nucl. Phys. 8:237 (1958).

12. O. Castanos, A. Frank, and P. Van Isacker, Phys. Rev. Lett. 54:263 (1984).

13. N. V. Zamfir and R. F. Casten, Phys. Lett., in press.

14. L. Wilets and M. Jean, Phys. Rev. 102:788 (1956).

15. R. F. Casten, "Nuclear Structure from a Simple Perspective," Oxford University Press, Oxford (1990), p. 195.

16. K. Heyde et al., Phys. Rev. C29:1420 (1984).

17. U. Neuneyer et al., Z. Phys. A336:2461 (1990); W. Lieberz et al., Z. Phys. A330:221 (1988); K. Schiffer et al., Z. Phys. A327:265 (1987); W. Lieberz et al., Phys. Lett. 240B:38 (1990); W. Zipper et ai., Nucl. Phys. A504:36 (1989).

18. P. von Brentano et al., in: Proc. Int. Conf. on High Spin Physics and Gamma-soft Nuclei, J. X. Saladin, R. A. Sorensen, and C. M. Vincent, eds., World Scientific, Singapore (1991), p. 344; P. von Brentano et al. in Proc. Predeal Intern. Summer School on Recent Advances in Nuclear Structure, World Scientific, Singapore, in press.

19. R. F. Casten, N. V. Zamfir, P. von Brentano, F. Seiffert, and W. Lieberz, to be pubiished.

20. H. G. Börner, J. Jolie, S. Robinson, R. F. Casten, and J. A. Cizewski, Phys. Rev. C42:R2271 (1990).

21. H. G. Börner et al., Phys. Lett. 215B:45 (1988); H. G. Börner, J. Jolie, F. Hoyler, and.S. Robinson in Contemporary Topics in Nuclear Structure Physics, R. F. Casten, A. Frank, S. Pittel, and M. Moshinsky, eds., World Scientific, Singapore (198E), p. 27: J. Jolie, S. J. Robinson, H. G. Börner, and P. Schillebeeckx in Spectroscopy of Heavy Nuclei, IOP Conf. Ser. 105:179 (1990).

22. J. Jolie, these proceedings.

23. H. G. Börner et al., Phys. Rev. Lett. 66:691 (1991).

24. H. G. Börner et al., Phys. Rev. C42:R2271 (1990).

25. J. P. Elliott, Proc. R. Soc. London, Ser. A245:128 (1958) and Ser. A245:562 (1958).

26. R. D. Ratna-Raju, j. P. Draayer, and K. T. Hecht, Nucl. Phy.s. A202:433 (1973).

27. J. P. Draayer and H. A. Naqui in High Spin Physics and Gamma-Soft Nuclei, J. X. Saladin, R. A. Sorensen, and C. M. Vincent, eds., World Scientific, Singapore (1991), p. 94; J. P. Draayer in Linderstanding the Variety of Nuclear Excitations, A. Covello, ed., World Scientific, Singapore (1991), p. 439. 
28. P. J. Nolan and P. J. Twin, Ann. Rev. Nucl. Part. Sci. $38: 533$ (1988), and references therein; W. Nazarewicz, P. J. Twin, P. Fallon, and J. D. Garrett, Phys. Rev. Lett. 64:1654 (1990); K. Zuber et al., Phys. Lett., to be published.

29. A. Gelberg, P. von Brentano, and R. F. Casten, J. Phys. G16:L143 (1990).

30. T. Byrski et al., Phys. Rev. Lett. 64:1650 (1990).

31. O. Castanos, P. O. Hess, J. P. Draayer, and P. Rochford, Nucl. Phys. A524:469 (1991); J. P. Draayer, Inst. Phys. Conf. Ser. 105:445 (1989). 